\title{
On Strongly Pettis Integrable Functions in Locally Convex Spaces
}

\author{
N. D. Chakraborty and SK. Jaker All
}

\begin{abstract}
Some characterizations have been given for the relative compactness of the range of the indefinite Pettis integral of a function defined on a complete finite measure space with values in a quasicomplete Hausdorff locally convex space. It has been shown that the indefinite Pettis integral has a relatively compact range if the functions is measurable by seminorm. Separation property has been defined for a scalarly measurable function and it has been proved that a function with this property is integrable by seminorm.
\end{abstract}

For a bounded function another characterization has been given for the relative compactness of the range of the indefinite Pettis integral. Dunford-Pettis-Phillips theorem has been generalized to locally convex spaces and as a corollary of this theorem some results which are valid for Banach spaces have been extended to locally convex spaces.

\section{INTRODUCTION}

The object of this paper is to study the relative compactness property of the range of the indefinite integral of a Pettis integrable function defined on a complete finite measure space with values in a quasicomplete locally convex space. It is wellknown that the range of the indefinite Pettis integral is always weakly relatively compact. Now the question whether it is relatively compact or not is a problem raised by B.J.Pettis many years ago. The answer was given in the negative by a counterexample constructed by Fremlin and Talagrand in their famous paper [13]. Stegall showed that the answer is affirmative if some restriction is imposed on the

1991 Mathematics Subject Classification: 28B05, 46G10.

Editorial Complutense. Madrid, 1993. 
underlying measure space-namely the measure space is perfect. Thereafter, many authors have studied the problem and have given different types of characterizations. Edgar [8] characterised this through a linear operator while Emmanuele and Musial did the same in a recent paper[11] through a bilinear functional associated with the function under consideration. All of these results were given in a Banach space setting. It has also been pointed in $[6, p .150]$ that the above problem has an affirmative answer for Banach spaces with Gelfand-Phillips property and some examples of this class of spaces have been presented, one of them being Banach spaces with weak* sequentially compact dual balls.

A. J. Pallares and G. Vera [23], [24] have made a detailed study of the problem and have generalized many of the above mentioned results to a quasi-complete locally convex space. They defined a Pettis integrable function to be strongly Pettis integrable if the range of the indefinite Pettis integral is relatively compact.

In this paper, we have studied strongly Pettis integrable functions and their connections with some linear operators associated with them in a locally convex space.

In Section 1, we have considered the functions which are not necessarily bounded and some equivalent conditions for the strong Pettis integrability have been established. Some of these, however, can be found in [24] though their proof is somewhat different. It has been shown that every Pettis integrable function taking values in a Gelfand-Phillips space is strongly Pettis integrable. Also we have proved that if a Pettis integrable function is measurable by seminorm, then it is strongly Pettis integrable which is a generalization of a well-known result in Banach space $[5$, p. 224]. The section has been closed with a result similar to one of A. Ionescu Tulcea [19].

In Section 2, the functions have been assumed to be bounded. The assumption enables us to introduce two new operators associated with the functions and their strong Pettis integrability has been studied through these operators. The well-known theorem of Dunford-Pettis-Phillips has been generalized to a locally convex space where the representing function is integrable by seminorm. As a corollary we have obtained Phillips' theorem and have also generalized two results of Geitz and Uhl [15] to locally convex spaces.

\section{NOTATIONS AND TERMINOLOGIES}

Throughout this paper $(\Omega, \Sigma, \mu)$ stands for a complete finite measure space. $X$ is a locally convex Hausdorff topological vector space assumed to be quasi-complete, in short, a locally convex space, with its topology $\mathscr{T}$. 
By $\mathscr{P}(X)$ we mean a fundamental collection of continuous seminorms on $X$ which determines the topology $\mathscr{T} . X^{\prime}$ denotes the topological dual of $X$. If the topology of $X$ (resp. $X^{\prime}$ ) is understood, it will be always assumed to be the original topology $\mathscr{F}$ (resp. strong topology $\beta\left(X^{\prime}, X\right)$ ). For $p \in \mathscr{P}(X), V_{p}$ represents the set $\{x \in X: p(x) \leqslant 1\}$ in $X$ and for $x_{0} \in X$ and $\varepsilon>0$, we write

$$
U_{p}\left(x_{0}, \varepsilon\right)=\left\{x \in X: p\left(x-x_{0}\right)<\varepsilon\right\}
$$

For a subset $A$ of $X, A^{0}$ denotes the polar of $A$ in $X^{\prime}$. The transpose of a continuous linear operator $T$ is denoted by $T^{*}$.

For the termilogies and properties used in the sequel concerning a locally convex space we refer to [18].

\section{STRONG PETTIS INTEGRABILITY}

1. Definitions. A function $f: \Omega \rightarrow X$ is said to be scalarly measurable (resp. scalarly integrable) if for each $x^{\prime} \in X^{\prime}$ the scalar function $x^{\prime} f$ is measurable (in $\mu$-almost everywhere) (resp. $x^{\prime} f \in L_{1}(\mu)$ ).

The function $f$ is said to be Pettis integrable if it is scalarly integrable and for each $E \in \Sigma$, there exists an $x_{E} \in X$ such that

$$
x^{\prime}\left(x_{E}\right)=\int_{E} x^{\prime} f d \mu
$$

for all $x^{\prime} \in \mathrm{X}^{\prime}$ and $x_{E}$ is called the Pettis integral of $f$ over $E$ and is denoted by

$$
x_{E}=P-\int_{E} f d \mu .
$$

In this case we obtain a countably additive, $\mu$-continuous vector measure $m_{f}: \Sigma \rightarrow X$ defined by

$$
m_{f}(E)=P-\int_{E} f d \mu
$$

for $E \in \Sigma$ and it is said to be induced by $f$. 
The function $f$ is said to be stronggly Pettis integrable if it is Pettis integrable and the range of the induced vector measure $m_{f}$, namely the set

$$
m_{f}(\Sigma)=\left\{P-\int_{E} f d \mu: E \in \Sigma\right\},
$$

is relatively compact in $X$ [24].

For a scalarly integrable function $f: \Omega \rightarrow X$ we define the linear operator $T_{f}: X^{\prime} \rightarrow L_{1}(\mu)$ by

$$
T_{f}\left(x^{\prime}\right)=x^{\prime} f
$$

for $x^{\prime} \in X^{\prime}$. It is easy to see that $f$ is Pettis integrable if and only if $T_{f}$ is weak* to weak continuous and if and only if $\emptyset f$ is Pettis integrable for each $\emptyset \in L_{\infty}(\mu)$. So, whenever $f$ is Pettis integrable we can define the linear operator

$$
\begin{aligned}
& I_{f}: L_{\infty}(\mu) \rightarrow X \text { by } \\
& I_{f}(\emptyset)=P-\int_{\Omega} \emptyset f d \mu
\end{aligned}
$$

for $\emptyset \in L_{\infty}(\mu)$. It can be shown that $I_{f}$ is continuous and

$$
\begin{aligned}
& T_{f}^{*}=I_{f} \text { and } \\
& I_{f}^{*}=T_{f} .
\end{aligned}
$$

For a scalarly integrable function $f: \Omega \rightarrow X$ we define the bilinear functional $Q_{f}$ on $X^{\prime} \times L_{\infty}(\mu)$ by

$$
Q_{f}\left(x^{\prime}, \varnothing\right)=\int_{\Omega} x^{\prime}(\varnothing f) d \mu
$$

for $x^{\prime} \in X^{\prime}$ and $\emptyset \in L_{\infty}(\mu)[11]$.

It is easy to verify that $f$ is Pettis integrable if and only if the bilinear functional $Q_{f}$ is separately continuous with respect to the product topology weak* $\times$ weak* of $X^{\prime} \times L_{\infty}(\mu)$.

Also we note that 


$$
Q_{f}\left(x^{\prime}, \emptyset\right)=<\varnothing, T_{f}\left(x^{\prime}\right)>=x^{\prime}\left(I_{f}(\varnothing)\right)
$$

for all $x^{\prime} \in X^{\prime}$ and $\emptyset \in L_{\infty}(\mu)$.

A subset $A$ of a locally convex space $X$ is said to be limited if

$$
\lim _{n} \sup _{x \in A}\left|x_{n}^{\prime} x\right|=0
$$

for each weak* null sequence $\left\{x_{n}^{\prime}\right\}$ in $X^{\prime}$.

A locally convex space $X$ is said to be a Gelfand-Phillips space whenever limited subsets of $X$ are relatively compact.

2. Lemma. The range of the induced vector measure of a Pettis integrable function $f: \Omega \rightarrow X$ is a limited subset of $X$.

Proof. Let $\left\{x_{n}^{\prime}\right\}$ be a weak* null sequence in $X^{\prime}$. Since $f$ is Pettis integrable, it can be proved, by an application of Vitali's convergence theorem, that the linear operator $T_{f}: X^{\prime} \rightarrow L_{1}(\mu)$ is weak* to $\|\cdot\|_{1}$ sequentially continuous. Hence the sequence $\left\{T_{f} x_{n}^{\prime}\right\}$, that is, $\left\{x_{n}^{\prime} f\right\}$ converges in $\|\cdot\|_{1}$ to zero in $L_{1}(\mu)$; in other words,

$$
\lim _{n}\left\|x_{n}^{\prime} f\right\|_{1}=0
$$

Now

$$
\begin{aligned}
\sup _{E \in \Sigma}\left|x_{n}^{\prime} m_{f}(E)\right| & =\sup _{E \in \Sigma}\left|\int_{E} x_{n}^{\prime} f d \mu\right| \\
& \leqslant \sup _{E \in \Sigma} \int_{E}\left|x_{n}^{\prime} f\right| d \mu \\
& \leqslant \int_{\Omega}\left|x_{n}^{\prime} f\right| d \mu \\
& =\left\|x_{n}^{\prime} f\right\|_{1}
\end{aligned}
$$

and hence

$$
\limsup _{n \in \Sigma}\left|x_{n}^{\prime} m_{f}(E)\right|=0
$$


which shows that the set $m_{f}(\Sigma)$ is a limited subset of $X$ and the lemma is proved.

3. Corollary. If $X$ is a Gelfand-Phillips space, then every Pettis integrable function $f: \Omega \rightarrow X$ is strongly Pettis integrable.

Note. If $X$ is a Banach space with a weak* sequentially compact dual ball, then every limited subset of $X$ is relatively compact $[4$, p. 238 , Ex.4(ii)] and hence every Pettis integrable function taking values in $X$ is strongly Pettis integrable.

The corresponding result for a locally convex space has been proved in Corollary 5(b).

We now give some characterizations for strong Pettis integrability of $f$ in terms of the operators $T_{f}$ and $I_{f}$ and the bilinear functional $Q_{f}$ as has been done in [8], [24], [23] and [11].

4. Theorem. Let $f: \Omega \rightarrow X$ be a Pettis integrable function. Then the following conditions are equivalent:

(1) $f$ is strongly Pettis integrable.

(2) For each $p \in \mathscr{Y}(X)$, the restriction of $T_{f}$ to $V_{p}^{o}$ is weak* to norm continuous.

(3) For each $p \in \mathscr{F}(X)$, the restriction of $Q_{f}$ to $V_{p}^{o} \times B_{L_{\alpha}(\mu)}$ is weak ${ }^{*} \times$ weak* continuous, where $B_{L_{\alpha}(u)}$ is the closed unit ball of $L_{\infty}(\mu)$.

(4) For each $p \in \mathscr{\mathscr { P }}(X)$, the set $\left\{x^{\prime} f: x^{\prime} \in V_{p}^{o}\right\}$ is norm compact in $L_{1}(\mu)$.

(5) For each $p \in \circlearrowright(X)$, every sequence $\left\{x_{n}^{\prime}\right\}$ in $V_{p}^{o}$ has a subsequence $\left\{x_{n_{k}}^{\prime}\right\}$ such that $\left\{x_{n}^{\prime} f\right\}$ converges $\mu$-almost everywhere.

(6) The restriction of $I_{f}$ to $B_{L_{x}(a)}$ is weak ${ }^{*}$ to. $\bar{T}$ continuous.

(7) $I_{f}$ is compact.

Proof. $(1) \Rightarrow(2)$. Let $p \in \mathscr{P}(X)$ and $\left\{x_{\alpha}^{\prime}\right\}$ be a net in $V_{p}^{o}$ converging to $x^{\prime}$ in the weak* topology. Since $V_{p}^{o}$ is equicontinuous in $X^{\prime}$ and $m_{f}(\Sigma)$ is relatively compact in $X$, the net $\left\{x_{a}^{\prime}\right\}$ converges uniformly on $m_{f}(\Sigma)$ to $x^{\prime}$. Now 
$\left\|T_{f}\left(x_{a}^{\prime}\right)-T_{f}\left(x^{\prime}\right)\right\|_{1}=\left|\left(x_{\alpha}^{\prime}-x^{\prime}\right) m_{f}\right|(\Omega) \leqslant 4$ sup $\left\{\left|\left(x_{\alpha}^{\prime}-x^{\prime}\right) m_{f}(E)\right|: E \in \Sigma\right\}$.

Consequently,

$$
\lim _{\alpha}\left\|T_{f}\left(x_{a}^{\prime}\right)-T_{f}\left(x^{\prime}\right)\right\|_{1}=0
$$

which gives (2).

(2) $\Rightarrow(3)$. Let $p \in \mathscr{S}^{\prime}(X)$ and $\left\{x_{\alpha}^{\prime}\right\}$ be a net in $V_{p}^{o}$ converging to $x^{\prime}$ in the weak* topology of $X^{\prime}$ and let $\left\{\varnothing_{\beta}\right\}$ be a net in $B_{L_{\infty}(u)}$ converging to $\emptyset$ in the weak* topology of $L_{\infty}(\mu)$. Then,

$$
\begin{aligned}
\left|Q_{f}\left(x_{\alpha}^{\prime}, \emptyset_{\beta}\right)-Q_{f}\left(x^{\prime}, \emptyset\right)\right| & =\left|<\emptyset_{\beta}, T_{f}\left(x_{\alpha}^{\prime}\right)>-<\varnothing, T_{f}\left(x^{\prime}\right)>\right| \\
& \leqslant\left|<\emptyset_{\beta}, T_{f}\left(x_{\alpha}^{\prime}-x^{\prime}\right)>\right|+\left|<\emptyset_{\beta}-\emptyset, T_{f}\left(x^{\prime}\right)>\right| \\
& \leqslant\left\|T_{f}\left(x_{\alpha}^{\prime}-x^{\prime}\right)\right\|_{1}+\mid<\emptyset_{\beta}-\emptyset, T_{f}\left(x^{\prime}\right)>1 .
\end{aligned}
$$

Since $T_{f}\left(x^{\prime}\right) \varepsilon L_{l}(\mu)$, we have

$$
\lim _{\alpha, \beta} Q_{f}\left(x_{\alpha}^{\prime}, \emptyset_{\beta}\right)=Q_{f}\left(x^{\prime}, \emptyset\right)
$$

and (3) follows.

(3) $\Rightarrow$ (4). Let $p \in$. $(X)$ and $\left\{x_{\alpha}^{\prime}\right\}$ be a net in $V_{p}^{o}$ and let $\left\{x_{\beta}^{\prime}\right\}$ be a subnet converging in the weak* topology of $X^{\prime}$ to an element $x^{\prime} \in V_{p}^{o}$. Let, for each $\beta$,

$$
E_{\beta}=\left\{\omega \in \Omega:\left(x_{\beta}^{\prime}-x^{\prime}\right) f(\omega) \geqslant 0\right\}
$$

so that $E_{\beta} \in \Sigma$ and

$$
\Omega \backslash E_{\beta}=\left\{\omega \in \Omega:\left(x_{\beta}^{\prime}-x^{\prime}\right) f(\omega)<0\right\} .
$$

We now put

$$
\emptyset_{\beta}=\chi_{\varepsilon_{\beta}}-\chi_{\mathrm{n} \backslash \varepsilon_{\beta}}
$$

Then it is easy to see that $\emptyset_{\beta} \in B_{L_{*}(\mu)}$ and

$$
\left\|\left(x_{\beta}^{\prime}-x^{\prime}\right)\right\|_{1}=\left|\int_{\Omega}\left(\left(x_{\beta}^{\prime}-x^{\prime}\right)(f)\right) \emptyset_{\beta} d \mu\right| .
$$

Let $\left\{\varnothing_{v}\right\}$ be a weak*-convergent subnet of $\left\{\emptyset_{\beta}\right\}$ with limit $\emptyset \in B_{L_{*}(\mu)}$. 
Now

$$
\begin{aligned}
\left\|\left(x_{v}^{\prime}-x^{\prime}\right)(f)\right\|_{1} & =\left|\int_{\Omega}\left(\left(x_{v}^{\prime}-x^{\prime}\right) f\right) \emptyset_{v} d \mu\right| \\
& =\left|Q_{f}\left(x_{v}^{\prime}-x^{\prime}, \emptyset_{v}\right)\right| .
\end{aligned}
$$

Not $\left\{x_{v}^{\prime}\right\}$, being a subnet of $\left\{x_{\beta}^{\prime}\right\}$, converges to $x^{\prime}$ in the weak* topology and so we have

$$
\lim _{\nu}\left\|\left(x_{\nu}^{\prime}-x^{\prime}\right) f\right\|_{1}=0
$$

which shows that the set $\left\{x^{\prime} f: x^{\prime} \in V_{p}^{o}\right\}$ is norm compact in $L_{1}(\mu)$.

(4) $\Rightarrow(5)$. Trivial.

$(5) \Rightarrow(4)$. Let $p \in \mathscr{P}(X)$. Let $\left\{x_{n}^{\prime}\right\}$ be a sequence in $V_{p}^{o}$. Then there exists a subsequence $\left\{x_{n_{k}}^{\prime}\right\}$ such that the sequence $\left\{x_{n_{k}}^{\prime} f\right\}$ converges $\mu$-almost everywhere. Also the sequence $\left\{x_{n_{k}}^{\prime} f\right\}$ is uniformly integrable [3, Lemma 1] or [22, p. 162]. Therefore an application of Vitali's convergence theorem implies that

$$
\left\|x_{n_{k}}^{\prime} f-x^{\prime} f\right\|_{1} \rightarrow 0
$$

which gives (4).

(4) $\Rightarrow(6)$. Let $\left\{\emptyset_{a}\right\}$ be a weak*-convergent net in $B_{L_{\infty}(\mu)}$ with limit $\emptyset$. Now for $p \in \mathscr{\mathscr { P }}(X)$,

$$
p\left(I_{f}\left(\emptyset_{\alpha}\right)-I_{f}(\emptyset)\right)=\sup _{x^{\prime} \in \mathbb{Y}_{p}^{o}}\left|<\emptyset_{\alpha}-\varnothing, x^{\prime} f>\right|
$$

and $B_{L_{\alpha}\{u\rangle}$ being equicontinuous, $\emptyset_{\alpha}$ converges uniformly to $\varnothing$ on the compact set $\left\{x^{\prime} f: x^{\prime} \in V_{p}^{o}\right\}$ and hence

$$
I_{f}\left(\emptyset_{\alpha}\right) \rightarrow I_{f}(\varnothing)
$$

in the $\mathscr{T}$-topology of $X$ and the result follows.

$(6) \Rightarrow(7)$. It is an immediate consequence of the fact that $B_{L_{x}(u)}$ is a neighbourhood of zero for the $\|\cdot\|_{\infty}$ topology as well as a weak* compact set in $L_{\infty}(\mu)$. 
(7) $\Rightarrow(1)$. It follows trivially from the fact that the set $\left\{\chi_{E}: E \in \Sigma\right\}$ is contained in $B_{L_{m}(\mu)}$.

Remark. If $X$ is complete, then Grothendieck's completeness theorem enables us to replace Pettis integrability by scalar integrability in order to prove the equivalences of the conditions (1), (2) and (3) in the above theorem.

5. Corollary. (a) A scalarly integrable function $f: \Omega \rightarrow X$ is strongly Pettis integrable if and only if $\emptyset f$ is strongly Pettis integrable for every $\emptyset \in L_{\infty}(\mu)$.

(b) If each equicontinuous set in $X^{\prime}$ is weak sequentially relatively compact, then every Pettis integrable function with values in $X$ is strongly Pettis integrable.

(c) If for a Pettis integrable function $f: \Omega \rightarrow X$, the operator $T_{f}$ is compact, then $f$ is strongly Pettis integrable.

If $X$ is metrizable, the converse is also true.

Proof. (a) and (b) are obvious from Theorem 4.

(c) The first part is trivial.

Conversely, it is given that $I_{f}$ is compact. Since $I_{f}^{*}=T_{f}$, the result follows from $[9, \mathrm{p}, 669$, Corollary 9.6.3].

We now consider the functions which are measurable by seminorm. For the definitions and properties of strong measurability and measurability by seminorm and strong integrability and integrability by seminorm of a function with values in a locally convex space we refer to [1].

It is well known $[27$, p. 65] that if a function $f: \Omega \rightarrow X$ is scalarly integrable, then for each $p \in \mathscr{P}^{\circ}(X)$,

$$
\bar{p}(f)=\sup _{x^{\prime} \in \mathbb{V}_{p}^{a}} \int_{\Omega}\left|x^{\prime} f\right| d \mu<\infty .
$$

The non-negative function $\bar{p}$ is called a Pettis seminorm over the space of all scalarly integrable functions with values in $X$. 
6. Theorem. If a Pettis integrable function $f: \Omega \rightarrow X$ is measurable by seminorm, then for each $p \in \mathscr{P}^{\prime}(X)$ there exists a sequence $\left\{f_{n}\right\}$ of simple functions such that

$$
\bar{p}\left(f_{n}-f\right) \rightarrow 0 .
$$

Proof. Let $p \in \mathscr{P}^{\prime}(X)$ and $\varepsilon>0$. It is sufficient to prove that there exists a simple function $f_{\varepsilon}: \Omega \rightarrow X$ such that

$$
\bar{p}\left(f_{\varepsilon}-f\right)<\varepsilon .
$$

Since $f$ is measurable by seminorm, without any loss of generality, we may assume that $f(\Omega)$ is separable for $p[1$, p. 84, Theorem 2.2] so that there exists a countable $p$-dense subset $\left\{x_{1}, x_{2}, \ldots\right\}$ in $f(\Omega)$.

Let

$$
\eta=\varepsilon / 3 \mu(\Omega) \text {, if } \mu(\Omega) \neq 0 .
$$

(If $\mu(\Omega)=0$, then the result is trivial).

Then the collection

$$
\left\{U_{p}\left(x_{n}, \eta\right): n=1,2, \ldots\right\}
$$

covers $f(\Omega)$. Let

$$
A_{n}=\left\{\omega \in \Omega: f(\omega) \in U_{p}\left(x_{n}, \eta\right)\right\} .
$$

It is easy to verify that $A_{n} \in \Sigma$ for each $n$. Let

$$
E_{1}=A_{1}
$$

and

$$
E_{n}=A_{n} \backslash \bigcup_{i=1}^{n-1} A_{i}, \text { for } n>1
$$

and let us define the function $g_{\varepsilon}: \Omega \rightarrow X$ by

$$
g_{\varepsilon}(\omega)=x_{n}
$$

if $\omega \in E_{n}$. It is obvious that $g_{\varepsilon}$ is measurable by seminorm and countablyvalued. Also for each $\omega \in \Omega$ 


$$
p\left(f(\omega)-g_{\varepsilon}(\omega)\right)<\eta
$$

so that

$$
\left|x^{\prime}\left(f(\omega)-g_{\varepsilon}(\omega)\right)\right|<\eta
$$

for all $x^{\prime} \in V_{p}^{o}$.

Now

$$
\begin{aligned}
\left|x^{\prime} g_{e}(\omega)\right| & \leqslant\left|x^{\prime}\left(g_{e}(\omega)-f(\omega)\right)\right|+\left|x^{\prime} f(\omega)\right| \\
& <\eta+\left|x^{\prime} f(\omega)\right|
\end{aligned}
$$

for all $\omega \in \Omega$ and $x^{\prime} \in V_{p}^{s}$. Hence for $E \in \Sigma$

$$
\int_{E}\left|x^{\prime} g_{\varepsilon}\right| d \mu \leqslant \eta \mu(E)+\int_{E}\left|x^{\prime} f\right| d \mu .
$$

So,

$$
\begin{aligned}
\sup _{x^{\prime} \in V_{p}^{p}} \int_{E}\left|x^{\prime} g_{\varepsilon}\right| d \mu & \leqslant \eta \mu(E)+\sup _{x^{\prime} \in V_{P}^{o}} \int_{\Omega}\left|x^{\prime}\left(\chi_{E} f\right)\right| d \mu \\
& \leqslant \eta \mu(\Omega)+\bar{p}\left(\chi_{E} f\right) .
\end{aligned}
$$

Putting

$$
\lambda(E)=\sup _{x^{\prime} \in v_{p}^{o}} \int_{E}\left|x^{\prime} g_{\varepsilon}\right| d \mu
$$

we have

$$
\lambda(E) \leqslant \varepsilon / 3+\bar{p}\left(\chi_{E} f\right) .
$$

Since $f$ is Pettis integrable, the family $\left\{x^{\prime} f: x^{\prime} \in V_{p}^{o}\right\}$ is uniformly integrable [22, p. 162]. Hence we can find a $\sigma>0$ such that

$$
\bar{p}\left(\chi_{E} f\right)<\varepsilon / 3
$$

for $E \in \Sigma$ with $\mu(E)<\sigma$. 
Now

$$
\mu(\Omega)=\sum_{n=1}^{\infty} \mu\left(E_{n}\right) .
$$

So there exists a positive integer $N_{\sigma}$ such that

$$
\sum_{n=N_{g}+1}^{\infty} \mu\left(E_{n}\right)<\sigma .
$$

Letting

$$
E_{\sigma}=\bigcup_{n=N_{\mathrm{a}}+1}^{\infty} E_{n}
$$

we have

$$
\mu\left(E_{\sigma}\right)<\sigma
$$

and hence

$$
\lambda\left(E_{o}\right)<2 \varepsilon / 3 .
$$

Finally, we define $f_{\varepsilon}: \Omega \rightarrow X$ by

$$
\begin{aligned}
f_{\varepsilon}(\omega) & =x_{n}, \text { if } \omega \in E_{n} \text { and } n \leqslant N_{\sigma} \\
& =0, \text { elsewhere. }
\end{aligned}
$$

Then $f_{\varepsilon}$ is evidently a simple function and measurable by semi-norm and

$$
\begin{aligned}
\bar{p}\left(f_{\varepsilon}-f\right) & \leqslant \sup _{x^{\prime} \in V_{p}^{o}} \int\left|x^{\prime}\left(f-g_{\varepsilon}\right)\right| d \mu+\sup _{x^{\prime} \in V_{p}^{o}} \int\left|x^{\prime}\left(g_{\varepsilon}-f_{\varepsilon}\right)\right| d \mu \\
& \leqslant \eta \mu(\Omega)+\sup _{x^{\prime} \in v_{p}^{o}}|| x^{\prime} g_{\varepsilon} \mid d \mu \\
& =\varepsilon / 3+\lambda\left(E_{\sigma}\right)<\varepsilon
\end{aligned}
$$

and the theorem is proved. 
7. Theorem. If a Pettis integrable function $f: \Omega \rightarrow X$ is measurable by seminorm, in particular strongly measurable, then it is strongly Pettis integrable.

Proof. Let $p \in \mathscr{P}(X)$. Then by the above theorem there exists a sequence $\left\{f_{n}\right\}$ of simple functions such that

$$
\bar{p}\left(f_{n}-f\right) \rightarrow 0
$$

Now, for $E \in \Sigma$,

$$
\begin{aligned}
p\left(\int_{E} f_{n} d \mu-m_{f}(E)\right) & \leqslant \sup _{x^{\prime} \in V_{p}^{o}} \int\left|x_{E}^{\prime}\left(f_{n}-f\right)\right| d \mu \\
& \leqslant \bar{p}\left(f_{n}-f\right) .
\end{aligned}
$$

Therefore,

$$
p\left(\int_{E} f_{n} d \mu-m_{f}(E)\right)
$$

converges to zero uniformly for $E \in \Sigma$. An appeal to [17, p. 22, Theorem 9] implies that $f$ is strongly Pettis integrable.

8. Corollary. A Pettis integrable function $f: \Omega \rightarrow X$ is strongly Pettis integrable in each of the following cases:

(i) $f$ is integrable by seminorm, in particular strongly integrable.

(ii) $f$ is weakly equivalent to a function $g: \Omega \rightarrow X$ which is measurable by seminorm.

(iii) $X$ is separable by seminorm.

(iv) $X$ is a locally convex Suslin space.

9. Definition. A scalarly measurable function $f: \Omega \rightarrow X$ is said to have the separation property over a set $K \subset X^{\prime}$ if the family $\left\{x^{\prime} f: x^{\prime} \in K\right\}$ 
of scalar-valued measurable functions has the separation property, that is, whenever $x_{1}^{\prime}, x_{2}^{\prime} \in K$ and $x_{1}^{\prime} f=x_{2}^{\prime} f \mu$-almost everywhere, then $x_{1}^{\prime} f(\omega)=$ $x_{2}^{\prime} f(\omega)$ for all $\omega \in \Omega$ [19].

The following theorem is analogous to a result of A.lonescu Tulcea $[19$, p. 177 , Theorem 3$]$ and it can be proved in a similar manner.

10. Theorem. Let $f: \Omega \rightarrow X$ be a scalarly measurable function. Then it is measurable by seminorm if and only if for every $p \in \mathscr{P}(X)$ there exists a set $\Omega_{p} \in \Sigma$ with $\mu\left(\Omega \backslash \Omega_{p}\right)=0$ such that the restriction of $f$ to $\Omega_{p}$ has the separation property over $V_{p}^{o}$.

Remark. With the help of the above theorem and a result of A. Ionescu Tulcea [19] or S.S. Khurana [20], an alternative proof of Theorem 7 can be given as follows:

Let $f$ be Pettis integrable and measurable by seminorm and let, for $p \in \mathscr{P}(X), \Omega_{p}$ be as in the above theorem. Let

$$
g_{p}=f \chi_{\Omega_{p}}
$$

and

$$
H_{p}=\left\{x^{\prime} f: x^{\prime} \in V_{p}^{o}\right\} .
$$

Clearly

$$
H_{p}=\left\{x^{\prime} g_{p}: x^{\prime} \in V_{p}^{o}\right\}
$$

and it has the separation property. Also it is convex and compact for the topology of pointwise convergence. Hence by [19, p. 171, Theorem 1] or $[20$, p. 388 , Theorem 1$], H_{p}$ is sequentially compact for the topology of pointwise convergence and the proof is complete by Theorem 4 .

11. Corollary. If a scalarly measurable function $f: \Omega \rightarrow X$ has the separation property over each equicontinuous set in $X^{\prime}$, then $f$ is measurable by seminorm and hence, if $f$ is Pettis integrable, then it is strongly Pettis integrable.

Proof. It follows immediately from Theorem 10 and Theorem 7. 


\section{BOUNDED FUNCTIONS}

We now consider a function $f: \Omega \rightarrow X$ which is bounded. In this case, $f$ is scalarly integrable whenever it is scalarly measurable. Moreover, $x^{\prime} f \in L_{\infty}(\mu)$ for each $x^{\prime} \in X^{\prime}$. Consequently a linear operator $u_{f}: X^{\prime}\left\llcorner\mathrm{L}_{\infty}(\mu)\right.$ can be defined by

$$
u_{f}\left(x^{\prime}\right)=x^{\prime} f
$$

for $x^{\prime} \in X^{\prime}$ and it can be proved that $f$ is Pettis integrable if and only if $u_{f}$ is weak* to weak* continuous and if and only if $\emptyset f$ is Pettis integrable for every $\emptyset \in L_{1}(\mu)$. Thus whenever $f$ is bounded and Pettis integrable we can define another linear operator $v_{f}: L_{1}(\mu) \rightarrow X$ by

$$
v_{f}(\emptyset)=P-\int_{\Omega} \varnothing f d \mu
$$

for $\emptyset \in L_{1}(\mu)$. It can be proved that $v_{f}$ is continuous and the restriction of $v_{f}$ to $L_{\infty}(\mu)$ coincides with the operator $I_{f}$.

Let us first give the following corollary of Theorem 4 .

12. Corollary. Let $f: \Omega \rightarrow X$ be a bounded Pettis integrable function. If for each $p \in \mathscr{P}^{\prime}(X)$ the set $\left\{x^{\prime} f: x^{\prime} \in V_{p}^{o}\right\}$ is weakly precompact in $L_{\infty}(\mu)$, then $f$ is strongly Pettis integrable.

Proof. Let $p \in \mathscr{P}(X)$ and $\left\{x_{n}^{\prime}\right\}$ be an arbitrary sequence in $V_{p}^{o}$. Now the set

$$
H_{p}=\left\{x^{\prime} f: x^{\prime} \in V_{p}^{o}\right\}
$$

is bounded and weakly precompact in $L_{\infty}(\mu)$. Since the identity map

$$
i: L_{\infty}(\mu) \rightarrow \mathrm{L}_{\infty}(\mu)
$$

is bounded, $\left\{x_{n}^{\prime}\right\}$ has a subsequence $\left\{x_{n_{k}}^{\prime}\right\}$ such that $\left\{x_{n_{k}}^{\prime} f\right\}$ converges $\mu$-almost everywhere $[25$, p. 528, Theorem 1]. Hence the corollary follows from Theorem 4.

Let us recall that a linear operator defined on a locally convex space into another such space is said to be a Dunford-Pettis operator if it is con- 
tinuous and transforms weakly relatively compact sets into relatively compact sets [14, p. 326].

13. Theorem. For a bounded Pettis integrable function $f: \Omega \rightarrow X$, the following statements are equivalent:

(1) The restriction of the operator $u_{f}$ to each equicontinuous set is weak ${ }^{*}$ to Mackey, that is, $\sigma\left(X^{\prime}, X\right)$ to $\mathscr{F}\left(L_{\alpha}(\mu), L_{l}(\mu)\right)$, continuous.

(2) $v_{f}$ is a Dunford-Pettis operator.

(3) The restriction of $v_{f}$ to $L_{\infty}(\mu)$ is compact.

(4) $f$ is strongly Pettis integrable.

(5) $\varnothing f$ is strongly Pettis integrable for every $\emptyset \in L_{l}(\mu)$.

Proof. (1) $\Leftrightarrow(2)$. Let $\left\{x_{a}^{\prime}\right\}$ be an arbitrary equicontinuous net in $X^{\prime}$ converging to an element $x^{\prime} \in X^{\prime}$ in the weak* topology and let $H$ be a weakly relatively compact set in $L_{1}(\mu)$. We note that

$$
<u_{f}\left(x_{a}^{\prime}\right), \emptyset>=x_{a}^{\prime}\left(v_{f}(\varnothing)\right)
$$

for each $\alpha$ and for each $\emptyset \in H$. Consequently the net $\left\{u_{f}\left(x_{\alpha}^{\prime}\right)\right\}$ converges uniformly on $H$ to $u_{f}\left(x^{\prime}\right)$ if and only if the net $\left\{x_{a}^{\prime}\right\}$ converges uniformly to $x^{\prime}$ on $v_{f}(H)$. Hence the equivalence is obtained by $[21$, p. 148 , Problem A].

(2) $\Rightarrow(3)$. It follows from the fact that the unit ball of $L_{\infty}(\mu)$ is weakly relatively compact in $L_{1}(\mu)$.

(3) $\Rightarrow(2)$. If $X_{p}$ is the Banach space associated with each $p \in \mathscr{P}(X)$ and $\pi_{p}$ is the canonical projection of $X$ into $X_{p}$, then it is easy to verify that whenever $v_{f}$ satisfies condition (3), the restriction of the operator $v_{f_{p}}: L_{1}(\mu) \rightarrow X_{p}$ to $L_{\infty}(\mu)$ defines a compact operator from $L_{\infty}(\mu)$ to $X_{p}$ where $f_{p}=\pi_{p} \circ f: \Omega \rightarrow X_{p}$ which is clearly Pettis integrable. Hence by [25, p. 530] $v_{f_{p}}$ is a Dunford-Pettis operator and, therefore, the result follows from the fact that a subset $K$ in $X$ is relatively compact iff $\pi_{p}(K)$ is relatively compact in $X_{p}$ for each $p \in \mathscr{P}(X)$. 
(3) $\Leftrightarrow(4)$. Since the restriction of $v_{f}$ to $L_{\infty}(\mu)$ coincides with the operator $I_{f}$, the equivalence follows from Theorem 4 .

$(4) \Leftrightarrow(5)$. It follows from Theorem 4 .

Now we are ready to extend a theorem of Dunford-Pettis-Phillips type to general locally convex spaces. In [26] E. Saab has extended the same for a particular class of locally convex spaces, called (BM)-spaces [10].

14. Theorem. If $T: L_{1}(\mu) \rightarrow X$ is a weakly compact linear operator, then there exists a function $f: \Omega \rightarrow X$ which is integrable by seminorm with essentially weakly relatively compact range such that

$$
T(\varnothing)=\int_{\Omega} \emptyset f d \mu
$$

for all $\emptyset \in L_{l}(\mu)$.

Proof. Let us define the vector measure $G: \Sigma \rightarrow X$ by

$$
G(E)=T\left(\chi_{E}\right)
$$

for $E \in \Sigma$. Since

$$
\left\{\chi_{E} / \mu(E): E \in \Sigma, \mu(E)>0\right\}
$$

is a bounded subset of $L_{1}(\mu)$ and $T$ is weakly compact, $G$ has a weakly relatively compact average range in $X$. Hence by [2, p. 245] we can find a function $f: \Omega \rightarrow X$ which is integrable by seminorm with its range contained $\mu$-almost everywhere in the average range of $G$ such that

$$
G(E)=\int_{E} f d \mu
$$

for $E \in \Sigma$. We now show that $f$ is the required function.

To do this, we note that for each simple function $\psi \in L_{1}(\mu)$

$$
T(\psi)=\int_{\Omega} \psi f d \mu .
$$


For an arbitrary function $\varnothing \in L_{1}(\mu)$, there exists a sequence $\left\{\emptyset_{n}\right\}$ of simple functions in $L_{1}(\mu)$ such that

$$
\left\|\varnothing_{n}-\varnothing\right\|_{1} \rightarrow 0
$$

and $T$ being continuous

$$
T\left(\emptyset_{n}\right) \rightarrow T(\varnothing)
$$

in $X$. Since $f$ is integrable by seminorm with essentially bounded range, $\emptyset f$ is integrable by seminorm and

$$
\lim _{n} \int_{\Omega} \emptyset_{n} f d \mu=\int_{\Omega} \emptyset f d \mu
$$

which implies that

$$
T(\emptyset)=\int_{\Omega} \emptyset f d \mu
$$

and the proof of the theorem is complete.

As an immediate consequence of the above theorem we have the following corollary.

15. Corollary. If for a bounded Pettis integrable function $f: \Omega \rightarrow X$, the operator $v_{f}$ is weakly compact, then $f$ is weakly equivalent to a function $g: \Omega \rightarrow X$ which is integrable by seminorm with essentially weakly relatively compact range. In particular, $f$ is strongly Pettis integrable.

16. Corollary. Let $f: \Omega \rightarrow X$ be a bounded Pettis integrable function. If for each $p \in \mathscr{P}(X)$, the set $\left\{x^{\prime} f: x^{\prime} \in V_{p}^{a}\right\}$ is weakly relatively compact in $L_{\infty}(\mu)$, then $f$ is weakly equivalent to a function $g: \Omega \rightarrow X$ which is integrable by seminorm. Consequently, $f$ is strongly Pettis integrable.

Proof. Since $f$ is bounded and Pettis integrable, the operator $v_{f}$ is continuous. Also it should be noted that

$$
v_{f}^{*}=u_{f} .
$$


According to the hypothesis, $u_{f}$ transforms equicontinuous subsets of $X^{\prime}$ into weakly relatively compact subsets of $L_{\infty}(\mu)$. So by [16, p. 95 , Corollary 1], $v_{f}$ transforms bounded subsets of $L_{1}(\mu)$ into weakly relatively compact subsets of $X$. Thus $v_{f}$ is weakly compact and the corollary follows from the previous one.

Remark. The above corollary is a partial generalization of a result of Geitz and Uhl [15, p. 76, Theorem 2].

17. Corollary. For a bounded scalarly integrable function $f: \Omega \rightarrow X$, the conclusions of the above corollary hold in each of the following cases:

(a) the operator $u_{f}$ is weak* to weak continuous.

(b) $f$ is Pettis integrable or $X$ is complete, and for each $p \in \hat{F}(X)$ the restriction of $u_{f}$ to $V_{p}^{o}$ is weak* to weak continuous.

Next we generalize Phillips' theorem [7, p. 671, Theorem 5.1] to locally convex spaces. For a similar result we refer to $[20$, p. 39 , Theorem 2].

18. Lemma. Let $Y$ and $Z$ be two Hausdorff locally convex spaces and let $\mathscr{F}_{1}$ be the topology of $Z$ and $Y^{\prime}$ be the dual of $Y$. If a linear operator $T: Y^{\prime} \rightarrow Z$ is Mackey to $\mathscr{T}_{1}\left(\right.$ that is, $\mathscr{T}\left(Y^{\prime}, Y\right)$ to $\left.\mathscr{F}_{1}\right)$ continuous, then it is weak* to weak continuous.

\section{Proof. Trivial.}

19. Corollary: If a scalarly measurable function $f: \Omega \rightarrow X$ has a weakly relatively compact range, then it is Pettis integrable and is weakly equivalent to a function which is integrable by seminorm and hence $f$ is strongly Pettis integrable.

Proof. Let $A$ be the closed absolutely convex hull of the range of $f$. Then $X$ being quasicomplete, $A$ is a balanced convex weakly compact set in $X$ and hence $A^{\circ}$ is a neighbourhood of zero in $X^{\prime}$ for the Mackey topo$\operatorname{logy} \mathscr{T}\left(X^{\prime}, X\right)$. Now for $x^{\prime} \in A^{v}$, we have 


$$
\left\|u_{f}\left(x^{\prime}\right)\right\|_{\infty} \leqslant 1
$$

which shows that $u_{f}$ is Mackey to norm continuous and hence by the above lemma, it is weak* to weak continuous and the result follows from Corollary 17 .

We finish with the following corollary which generalizes another result of Geitz and Uhl [15, p. 78, Theorem 4].

20. Corollary. Let $X$ be complete and $f: \Omega \rightarrow X$ a bounded scalarly measurable function. If for each $p \in \mathscr{P}^{\gamma}(X)$, the set $\left\{x^{\prime} f: x^{\prime} \in V_{p}^{o}\right\}$ is weakly relatively compact in $B(\Omega, \Sigma)$, the space of all scalar valued bounded $\Sigma$ measurable functions on $\Omega$ equipped with the supnorm, then $f$ is weakly equivalent to a function which is integrable by seminorm and consequently $f$ is strongly Pettis integrable.

Proof. Let $p \in \mathscr{P}^{\circ}(X)$ and $\left\{x_{\alpha}^{\prime}\right\}$ be a weak*-convergent net in $V_{p}^{o}$. The set

$$
H_{p}=\left\{x^{\prime} f: x^{\prime} \in V_{p}^{o}\right\}
$$

is weakly relatively compact in $B(\Omega, \Sigma)$ and $f$ being bounded, $H_{p}$ is uniformly bounded. Hence, by $[12$, p. 78 and p. 80] the pointwise topology and the weak topology of $B(\Omega, \Sigma)$ coincide on $H_{p}$. Since the net $\left\{x_{\alpha}^{\prime} f\right\}$ converges weakly in $B(\Omega, \Sigma)$ and since

$$
\left(L_{\infty}(\mu)\right)^{\prime} \subset(B(\Omega, \Sigma))^{\prime}
$$

$\left\{x_{a}^{\prime} f\right\}$ converges weakly in $L_{\infty}(\mu)$. This implies that the restriction of $u_{f}$ to $V_{p}^{o}$ is weak* to weak continuous and the corollary follows from Corollary 17.

\section{Acknowledgements}

The authors gratefully acknowledge the comments and suggestions of the anonymous referee, which helped them to improve the presentation of the paper to a great extent. He pointed out the notion of Gelfand-Phillips spaces in connection with strong Pettis integrability and this helped the authors to incorporate Lemma 2 and Corollary 3 in the revised version of the paper. 


\section{References}

[1] C. BLONDIA: Integration in locally convex spaces, Simon Stevin, 55 (1981), 81-102.

[2] C. Blondia: A Radon-Nikodym theorem for vector valued measures, Bull. Soc. Math. Belg. Ser. B, 33 (1981), 231-249.

[3] S. D. Chatterjee: Sur l'integràbilité de Pettis, Math. Z., 136 (1974), 5358.

[4] J. DIESTEL: Sequences and series in Banach spaces, GTM-Vol. 92, Springer-Verlag, New York, 1984.

[5] J. Diestel and J. J. Uhl, Jr.: Vector measures, Math Surveys 15, American Mathematical Society, Providence, R. I., 1977.

[6] J. Diestel and J. J. UHL, Jr.: Measure Theory and its Applications, Lect. Notes in Math. Vol. 1033, Springer-Verlag, Berlin, 1983.

[7] G. A. EDGAR: Measurability in a Banach space, Indiana Univ. Math. J., 26 (1977), 663-677.

[8] G. A. EdGAR: Measurability in a Banach space, II, Indiana Univ. Math. J. 28 (1979), 559-579.

[9] R. E. EDwaRDs: Functional Analysis, Theory and Applications, Holt, Rinehart and Winston, New York, 1965.

[10] L. EGGHE: On the Radon-Nikodym property and related topics in locally convex spaces, Lecture Notes in Math. Vol. 645, Springer-Verlag (1978).

[11] G. EMMANUELE and K. Musial: Weak precompactness in the space of Pettis integrable functions, J. Math. Anal. Appl. 148 (1990), 245-250.

[12] K. FLORET: Weakly compact sets, Lecture Notes in Math. Vol. 801, Springer-Verlag (1980).

[13] D. Fremlin and M. TAlagrand: A decomposition theorem for additive set functions, with applications to Pettis integrals and ergodic means, Math. $Z$. 168 (1979), 117-142.

[14] A. Garcta and J. Gómez: Dunford-Pettis property, Arch. Math. 49 (1987), 326-332.

[15] R. F. GEITZ and J. J. UHL, Jr.: Vector valued functions as families of scalar valued functions, Pacific J. Math. 95 (1981), 75-83.

[16] A. GROTHENDIECK: Topological vector spaces, Gordon and Breach, New York (1973).

[17] J. HoffMAN-JoRgenson: Vector measures, Math. Scand, 28 (1971), 5-32.

[18] J. HoRvATH: Topological vector spaces and distributions, Addison-Wesley (1966).

[19] A. IONESCU TULCEA: On pointwise convergence, compactness and equicontinuity II, Adv. Math. 12 (1974), 171-177.

[20] S. S. Khurana: Pointwise compactness and measurability, Pacific J. Math. 83 (1979), 387-39I.

[21] J. L. Kelley and I. NAmIoKA: Linear Topological spaces, Van Nostrand, New York, 1963.

[22] K. Musial: Vitali and Lebesgue convergence theorems for Pettis integral in locally convex spaces, Atti Sem. Math. Fis. Univ. Modena, 35 (1987), 159-166. 
[23] A. J. Pallakés Ruiz: El espacio de las funciones integrables Pettis con recorrido relativamente compacto, Actas X Jornadas Hispano-Lusas de Matemáticas-Sección III Universidad de Murcia, 1 (1985), 385-391.

[24] A. J. Pallarés and G. Vera: El recorrido de la integral indefinida de Pettis, Rev. Real Acad. Cienc. Exac. Fis. Nat. de Madrid, 80 (1986), 121131.

[25] L. H. RIDDLE, E. SAAB and J. J. UHL, Jr.: Sets with the weak Radon-Nikodym Property in dual Banach spaces, Indiana Univ. Math. J. 32 (1983), 527-541.

[26] E. SAAB: On the Radon-Nikodym property in a class of locally convex spaces, Pacific J. Math. 75 (1978), 281-291.

[27] G. E. F. THOMAs: Integration of functions with values in locally convex Suslin spaces, Trans. Amer. Math. Soc. 212 (1975), 61-81.

Department of Mathematics

University of Burdwan

Burdwan - 713104

Recibido: 27 de marzo de 1991

West Bengal, India 